\title{
A Phase II Multi-Center, Non-Randomized, Parallel Group, Non-Inferiority Study to Compare the Efficacy of No Radioactive Iodine Remnant Ablation to Remnant Ablation Treatment in Low- to Intermediate-Risk of Papillary Thyroid Cancer: The MOREthyroid Trial Protocol
}

Eun Kyung Lee ${ }^{1}$, You Jin Lee ${ }^{1}$, Young Joo Park ${ }^{2}$, Jae Hoon Moon ${ }^{3}$, Ka Hee Yi ${ }^{4}$, Koon Soon Kim ${ }^{5}$ Joo Hee Lee ${ }^{5}$, Sun Wook $\mathrm{Cho}^{2}$, Jungnam Joo ${ }^{6}$, Yul Hwangbo ${ }^{1}$, Sujeong Go ${ }^{1}$, Do Joon Park ${ }^{2}$

${ }^{1}$ Center for Thyroid Cancer, National Cancer Center, Goyang; ${ }^{2}$ Department of Internal Medicine, Seoul National University Hospital, Seoul; ${ }^{3}$ Department of Internal Medicine, Seoul National University Bundang Hospital, Seongnam; ${ }^{4}$ Department of Internal Medicine, Seoul Metropolitan Government Seoul National University Boramae Medical Center, Seoul; ${ }^{5}$ Department of Internal Medicine, Chungnam National University College of Medicine, Daejeon; ${ }^{6}$ Cancer Biostatistics Branch, Research Institute for National Cancer Control and Evaluation, National Cancer Center, Goyang, Korea

Background: Radioactive iodine (RAI) remnant ablation is recommended in patients with papillary thyroid cancer (PTC) and extrathyroidal extension or central lymph node metastasis. However, there exists little evidence about the necessity of remnant ablation in PTC patients with low- to intermediate-risk, those have been increasing in recent decades.

Methods: This multicenter, prospective, non-randomized, parallel group clinical trial will enroll 310 eligible patients with low- to intermediate-risk of thyroid cancer. Inclusion criteria are patients who recently underwent total thyroidectomy for PTC with 3 or less tumors of size $1 \leq$ to $\leq 2 \mathrm{~cm}$ with no microscopic extension and N0/x, or size $\leq 2 \mathrm{~cm}$ with microscopic extension and/or N1a (number of lymph node $\leq 3$, size of tumor foci $\leq 0.2 \mathrm{~cm}$, and lymph node ratio $<0.4)$. Patients choose to undergo RAI ablation $\left({ }^{131} \mathrm{I}\right.$, dose $1.1 \mathrm{GBq}$ ) or diagnostic whole-body scan (DxWBS) $\left({ }^{131} \mathrm{I}\right.$ or ${ }^{123} \mathrm{I}$, dose 0.074 to $0.222 \mathrm{GBq}$ ), followed by subsequent measurement of stimulated thyroglobulin (sTg) within 1 year. Survey for quality of life (QOL) will be performed at baseline and at 1 year after follow-up. The total enrollment period is 5 years, and patients will be followed up for 1 year. The primary endpoint is the non-inferiority of surgery alone to surgery with ablation in terms of biochemical remission (BCR) rate (sTg $\leq 2 \mathrm{ng} / \mathrm{mL}$ ) without evidence of structural recurrence. The secondary endpoint was the difference of QOL.

Conclusion: This study will evaluate whether surgery alone achieves similar BCR and improved QOL compared to RAI ablation in patients with low- to intermediate-risk PTC within 1 year.

Keywords: Iodine-131; Quality of life; Thyroid cancer, papillary; Prospective studies

Received: 16 April 2020, Revised: 5 June 2020, Accepted: 6 July 2020

Corresponding author: Young Joo Park

Department of Internal Medicine, Seoul National University College of Medicine, 101 Daehak-ro, Jongno-gu, Seoul 03080, Korea

Tel: +82-2-2072-4183, Fax: +82-2-764-2199, E-mail: yjparkmd@snu.ac.kr
Copyright $\odot 2020$ Korean Endocrine Society

This is an Open Access article distributed under the terms of the Creative Commons Attribution Non-Commercial License (https://creativecommons.org/ licenses/by-nc/4.0/) which permits unrestricted non-commercial use, distribution, and reproduction in any medium, provided the original work is properly cited. 


\section{INTRODUCTION}

Radioactive iodine therapy (RAI) is a standard adjuvant treatment to prevent recurrence and ablate remnant thyroid tissues in patients with differentiated thyroid cancer (DTC) [1]. The dose of RAI varies from 1.1 to $7.4 \mathrm{GBq}$ based on the disease burden assessed by risk stratification, as described in several guidelines $[1,2]$. Recently, two well-designed prospective randomized trials reported that the efficacy of low-dose ablation $(1.1 \mathrm{GBq})$ was not inferior to that of high-dose ablation (>3.7 GBq) for patients with low-risk DTC $[3,4]$. However, the necessity of RAI in patients with low-risk DTC has not been primarily studied. If RAI is not indicated, the role of total thyroidectomy can be specified. Therefore, the efficacy and adverse events of RAI should be fundamentally assessed.

We propose a prospective, non-randomized, controlled clinical trial (the Minimize radioactive iodine ablation Of REmnant thyroid in differentiated thyroid cancer [MOREthyroid] trial) to assess whether RAI can induce biochemical remission (BCR). The basic question of the MOREthyroid study design was whether low-dose (1.1 GBq) RAI remnant ablation has marked efficacy with an acceptable quality of life (QOL), relative to diagnostic whole-body scan (DxWBS) surveillance to detect the small volume of iodine-avid metastases.

This proposed trial is designed to assess whether, in patients with low- to intermediate-risk DTC, surgery alone achieves a comparative BCR and an improved QOL compared to RAI remnant ablation within 1 year.

\section{METHODS}

\section{Study design}

The MOREthyroid trial is an investigator-initiated, open-label, parallel-assigned, multicenter, non-randomized trial (Table 1, Fig. 1). The trial protocol follows all recommendations of the Standard Protocol Items: Recommendations for Interventional Trials Statement (SPIRIT) 2013 [5]. Patients are to be recruited in five academic hospitals selected as study centers (National Cancer Center [NCC], Seoul National University Hospital, Seoul National University Bundang Hospital, Seoul Metropolitan Government Seoul National University Boramae Medical Center, and Chungnam National University Hospital).

\section{Participants}

Patients will be assigned to one of the two arms based on a 1:1 allocation. The Institutional Review Board (IRB) of NCC has approved this study protocol (IRB No. NCCCTS13-671). Moreover, this study has been approved by the local ethics committee of each study center (Seoul National University Hospital: IRB No. 1402-093-559, Seoul National University Bundang Hospital: IRB No. B-1405-250-401, Seoul Metropolitan Government Seoul National University Boramae Medical Center: IRB No. 26-2014-56, and Chungnam National University Hospital: IRB No. 2014-04-028). Written informed consent will be obtained from all participants prior to enrollment. This trial has been registered in the database of clinicaltrials.gov (NCT02418247). Patients or the public were not involved in the design, or conduct, or reporting, or dissemination plans of our research.

\section{Study population and eligibility criteria}

The eligibility for study inclusion are: diagnosis of papillary thyroid cancer (PTC); total thyroidectomy within the 6 months preceding the study enrollment; single or multifocal tumors $(\leq 3)$ of size $1 \leq$ to $\leq 2 \mathrm{~cm}$ without microscopic extension and $\mathrm{N} 0 / \mathrm{x}$, or size $\leq 2 \mathrm{~cm}$ with microscopic extension (less than strap muscle) and/or N1a (number of lymph nodes $\leq 3$, size of tumor foci $\leq$

Table 1. Summary of the Visit Schedule and Assessed Parameters at Each Time Point

\begin{tabular}{|c|c|c|c|c|c|}
\hline Variable & Baseline $^{\mathrm{a}}$ & $3 \mathrm{mo}$ & $6 \mathrm{mo}$ & $9-15 \mathrm{mo}$ & Every 1 yr \\
\hline \multicolumn{6}{|l|}{ RAI } \\
\hline RAI group & & $\mathrm{I}^{131} 1.1 \mathrm{GBq}$ & & $\mathrm{I}^{131} 1.1 \mathrm{GBq}$ & \\
\hline No-RAI group & & $\mathrm{I}^{123}$ or $\mathrm{I}^{131} 2-6 \mathrm{mCi}$ & & None & \\
\hline $\mathrm{Tg} / \mathrm{TgAb} / \mathrm{TSH}$ & & $\stackrel{+}{+}$ & $\stackrel{+}{+}$ & $\stackrel{+}{\stackrel{+}{(\text { stimulated })^{\mathrm{b}}}}$ & $\stackrel{+}{+}$ \\
\hline Neck USG & & & + & & + \\
\hline QOL questionnaire & + & + & & + & \\
\hline
\end{tabular}

RAI, radioactive iodine; Tg, thyroglobulin; TgAb, anti-thyroglobulin antibody; TSH, thyroid stimulating hormone; USG, ultrasonography; QOL, quality of life.

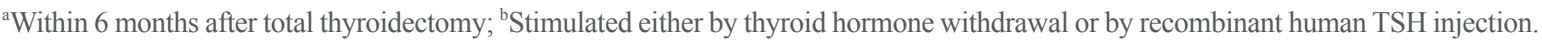




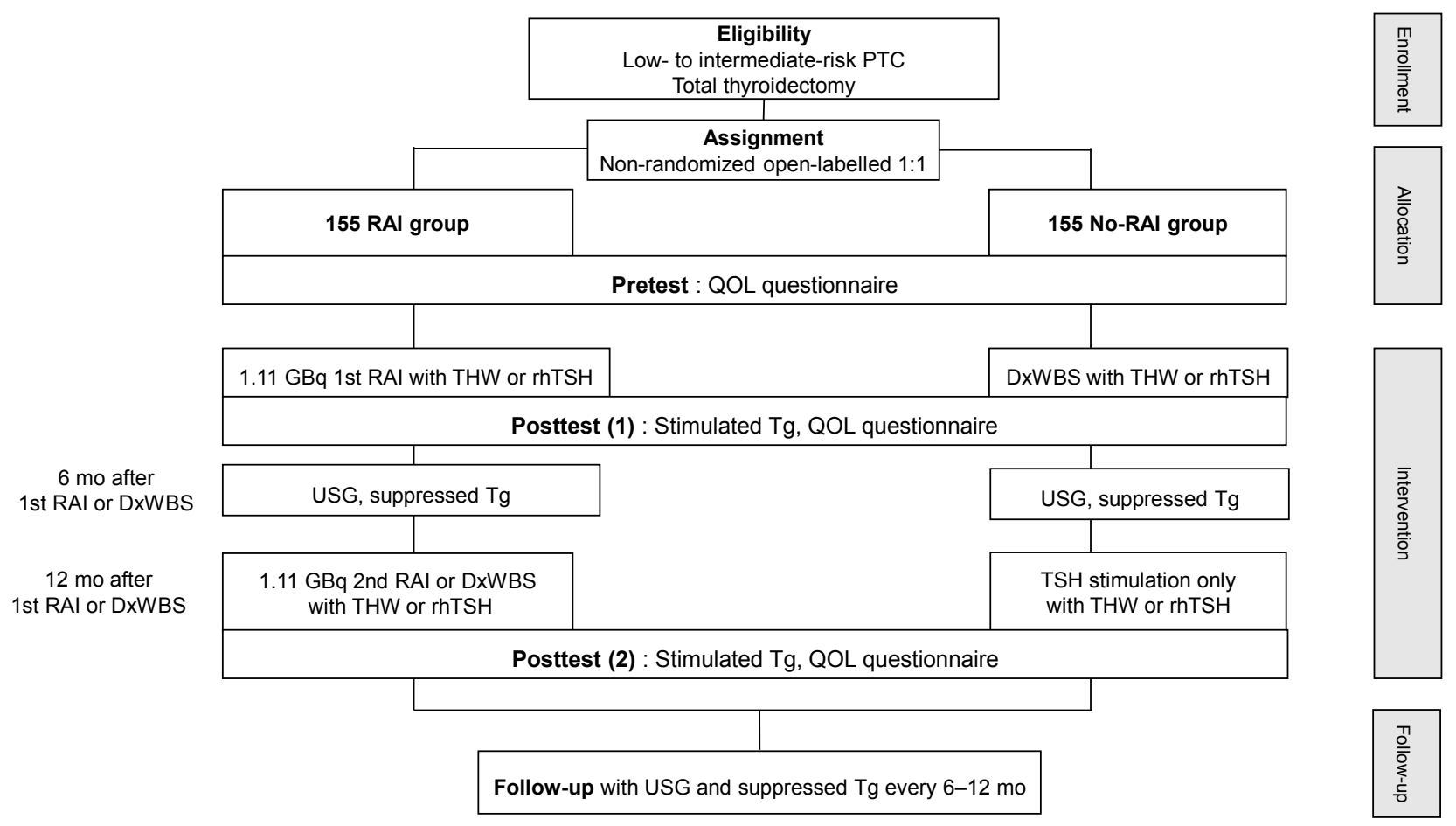

Fig. 1. Flow chart of study. PTC, papillary thyroid cancer; RAI, radioactive iodine; QOL, quality of life; THW, thyroid hormone withdrawal; rhTSH, recombinant human thyroid stimulating hormone; DxWBS, diagnostic whole body scan; Tg, thyroglobulin; USG, ultrasonography.

\begin{tabular}{|c|c|c|c|c|c|c|}
\hline \multirow[b]{3}{*}{ Tumor } & \multicolumn{6}{|c|}{ N stage } \\
\hline & \multirow{2}{*}{\multicolumn{2}{|c|}{$\mathrm{N} 0 / \mathrm{Nx}$}} & \multicolumn{2}{|c|}{ N1a } & \multirow[b]{2}{*}{ N1b } & \multirow[b]{2}{*}{$\begin{array}{c}\text { Any } \\
\text { N }\end{array}$} \\
\hline & & & $\begin{array}{c}\text { Metastatic } \\
\text { LNs } \leq 3 \text { and } \\
\text { tumor foci } \\
\leq 0.2 \mathrm{~cm}\end{array}$ & $\begin{array}{l}\text { Metastatic } \\
\text { LNs }>3 \text { or } \\
\text { tumor foci } \\
>0.2 \mathrm{~cm}\end{array}$ & & \\
\hline Size, $\mathrm{cm}$ & $<1$ & $\geq 1, \leq 2$ & $\leq 2$ & Any & Any & $>2$ \\
\hline \multicolumn{7}{|l|}{ ETE } \\
\hline Negative & $x$ & ० & $\circ$ & $x$ & $x$ & $x$ \\
\hline Microscopic & $\circ$ & ○ & ○ & $x$ & $\times$ & $\times$ \\
\hline
\end{tabular}

LN, lymph node; ETE, extrathyroidal extension.

$0.2 \mathrm{~cm}$, and lymph node ratio $<0.4$ ) according to the recommendations of the American Joint Committee for Cancer (AJCC), seventh edition; age 18 to 79 years; and willingness to provide written informed consent for voluntary study participation. The histological enrollment criteria summarized in Table 2 will be evaluated by individual investigators without central review.

The criteria for exclusion include aggressive PTC (tall cell, columnar cell, diffuse sclerosing, solid/trabecular, and insular variants), follicular thyroid cancer, poorly DTC, medullary thy- roid cancer, or anaplastic thyroid cancer; tumor size $>2 \mathrm{~cm}$; gross extension (strap muscle or more); a large volume of lymph node metastasis (tumor foci bigger than $0.2 \mathrm{~cm}$, metastasis to more than three lymph nodes, or lateral neck lymph node metastasis (pN1b); distant metastasis diagnosed at the time of enrollment; remote history of surgery for thyroid cancer; history of cervical external beam radiation therapy; diagnosis of other malignancies within 5 years preceding the study enrollment; renal insufficiency (estimated glomerular filtration rate $<30 \mathrm{~mL} / \mathrm{min} / 1.73 \mathrm{~m}^{2}$ ); and pregnancy or breastfeeding.

\section{Allocation}

The Web-based clinical trial management system (eVelos System, Velos Inc., Fremont, CA, USA; http://eresearch.ncc.re.kr/ velos/jsp/ereslogin.jsp) at the Clinical Research Coordination Center (CRCC), NCC, Korea, will be used to coordinate the study and undertake data analysis. After an eligible patient provides written informed consent, the patient will be registered in the system and allocated to one of the two study groups based on patient preference: DxWBS only or RAI ablation, undertaken as per the guidelines of the American Thyroid Association [1] and Korean Thyroid Association [2].

All registered subjects will undergo neck ultrasonography (US) 
at least once, with an interval of 6 months to 1 year, to detect locoregional recurrence; stimulated serum thyroglobulin ( $\mathrm{Tg}$ ) will be measured twice, with an interval of 3 months to 1 year to determine BCR; and QOL will be assessed by a self-reported questionnaire and completed in a face-to-face interview at the time of stimulated thyroglobulin (sTg) measurement (Table 1).

\section{Interventions}

\section{Experimental arm: no-RAI group}

The experimental arm (no-RAI group) comprises non-ablated patients, who would instead undergo a diagnostic scan. After study enrollment, a DxWBS with the first measurement of sTg would be done within 6 months after the initial surgery (total thyroidectomy). If abnormal uptake in regions other than the neck is detected on the first postoperative scan, the subjects will be treated by an appropriate treatment modality (low-/high-dose RAI, surgery, or radiation therapy) after imaging studies such as computed tomography (CT) or magnetic resonance imaging. At the next visit, suppressed Tg and neck US will be carried out. In case of a suspicious lesion on US, the patient will undergo USguided aspiration cytologic evaluation and/or measurement of washout Tg. The second sTg will be measured between 3 months and 1 year after the initial sTg measurement and DxWBS.

\section{Control arm: RAI group}

The control arm (RAI group) is the ablated group and comprises patients who will undergo low-dose RAI ablation with the dose of $1.1 \mathrm{GBq}$. After enrollment, the first RAI and measurement of sTg would be undertaken within 6 months after the total thyroidectomy, similarly as in the experimental group. In case of abnormal uptake at sites other than the neck, the subjects will be treated by an appropriate treatment modality. A second RAI would be conducted between 3 months and 1 year after the initial RAI. In case of no or faint uptake in the neck and undetectable sTg, a second RAI can be substituted with only sTg measurement after thyroid hormone withdrawal.

\section{Outcomes}

\section{Primary outcome}

The primary endpoint of the MOREthyroid trial is a comparison of the BCR rates of the no-RAI and RAI groups. To assess the $\mathrm{BCR}$ rate, the criteria are: (1) stimulated serum $\mathrm{Tg}<2 \mathrm{ng} / \mathrm{mL}$ at the second measurement; (2) stimulated anti-Tg antibodies (TgAbs) $\leq 100$; (3) no evidence of structural residual or recurrent disease on neck US or other imaging modalities; and (4) no uptake other than in the thyroid bed in whole body scan. Ade- quate sTg is defined as a serum Tg level measured when the serum thyroid stimulating hormone (TSH) level was more than 30 $\mathrm{mIU} / \mathrm{L}$ at 4 weeks of withdrawal with 2 weeks of either triiodothyronine (T3) replacement or recombinant human TSH injections. T3 will be discontinued 2 weeks prior to RAI. The serum TSH levels will be measured by an immunoradiometric assay (IRMA) with a commercial kit (Immunotech, Marseille, France). The serum $\mathrm{Tg}$ and anti-TgAb levels will be measured with IRMA and a commercial radioimmunoassay kit, respectively (BRAHMS, Hennigsdorf, Germany and DIAsource, Nivelles, Belgium). The reference ranges for $\mathrm{TSH}, \mathrm{Tg}$, and $\mathrm{TgAb}$ are 0.17 to $4.05 \mathrm{mU} / \mathrm{L}, 0$ to $40 \mathrm{ng} / \mathrm{mL}$, and 0 to $60 \mathrm{U} / \mathrm{mL}$ for BRAHMS and 0 to $70 \mathrm{U} / \mathrm{mL}$ for DIAsource, respectively. The $\mathrm{TgAb}$ concentration was considered positive when it exceeded $100 \mathrm{U} / \mathrm{mL}$.

\section{Secondary outcomes}

The secondary endpoints of this trial include recurrence-free survival, QOL, and disease-specific survival. QOL will be assessed by the validated Korean version of the thyroid-specific QOL questionnaire (KT-QoL) [6,7]. The QOL questionnaire evaluates four domains: physical, psychological, social and spiritual aspects. The score ranges from 1 to 10 . QOL will be assessed at least three times: the first assessment will be conducted at the time of intervention assignment (RAI or no-RAI) immediately after surgery, while the second and third QOL surveys are performed during the first and second measurements of sTg.

Disease-specific and recurrence-free survival will be assessed at least 5 years after the last enrollment. Patients showing abnormal RAI uptake will undergo additional imaging tests (CT or positron emission tomography) and be treated with appropriate treatment (surgery, high dose RAI therapy, etc.). If there is evidence of a structural disease after imaging, this is defined as a recurrence, according to the investigators' opinion. US will be performed regularly at the patient's visit to check for recurrence.

\section{Sample-size calculation}

The current study is designed with a non-inferiority design to show that the remission rate of the no-RAI group is non-inferior to that of the RAI group. The sample size was based on the difference in the BCR rate between subjects who did or did not receive low-dose RAI. Initially, the 1-year BCR rates for the RAI and non-RAI groups were assumed to be $80 \%$. The maximum permissible difference was determined as $10 \%$ points (non-inferiority margin of $10 \%$ ), which means that the remission rate of the non-RAI group should not be less than $70 \%$. To show $80 \%$ 
power and 2.5\% one-sided type I error, 250 patients are needed per study group. Considering a drop-out rate of $10 \%$, we determined that 278 subjects should be enrolled in each study group. An interim analysis after 287 subjects were enrolled found that $92 \%$ of the study sample had BCR at the first sTg measurement (Supplemental Table S1). Therefore, the required number of samples was re-calculated, and it was determined that a total of 310 patients who had completed the study protocol would be needed in the final analysis to generate adequate statistical power.

\section{Data management}

All patient data will be maintained in electronic case report forms (eCRFs) in a Web-based central platform (eVelos System) at the CRCC. The management team at the CRCC will review the eCRFs, and regularly send queries to each investigative site. Data monitoring will be undertaken through site visits, and the data will be managed and analyzed according to the study protocol.

\section{Safety assessment}

Any serious adverse events will be documented in the medical records as well as in the eCRF and reported to the IRB by the responsible investigator, in accordance with the local regulations.

\section{Statistical analysis}

The unpooled Z-test will be applied to assess the non-inferiority of non-RAI to RAI (Supplemental Table S2) [8]. Analyses of recurrence-free survival will be conducted on the Cox proportional hazards model, and Kaplan-Meier curves will be used for the estimation of survival curve. Categorical variables will be analyzed by the Pearson's chi-square test or Fisher's exact test, and continuous variables will be evaluated by the Student's $t$ test or an appropriate non-parametric method. $P$ values $<0.05$ will be considered statistically significant.

\section{Ethics and dissemination}

The protocol has been approved by the ethics committee of National Cancer Center (NCCCTS13671). The results of this trial will be disseminated through peer-reviewed publications and conferences. Data are available upon reasonable request.

\section{Trial status}

The trial is currently ongoing without further enrollment. The first patient was included on June 2013.

\section{DISCUSSION}

In order to define the group of low- to intermediate-risk patients who do not usually require remnant ablation because of a low risk of recurrence of less than $5 \%$, we retrospectively analyzed $\mathrm{BCR}$ rates by using the prospectively constructed clinicopathological database of the NCC Center for Thyroid Cancer. Patients with PTC who underwent total thyroidectomy and RAI therapy, with primary tumors smaller than $4 \mathrm{~cm}$, no or microscopic extrathyroidal extension, up to three multifocality, three or more dissected central lymph nodes, up to five metastatic lymph nodes, no lateral lymph node metastasis, and negative resection margin were selected. Of the 1,495 eligible population, we excluded 845 patients excluded due to incomplete ablation, insufficient TSH stimulation, or positive Tg antibody. The treatment outcome of 650 subjects was analyzed.

Table 3. Biochemical Results of Low- to Intermediate-Risk Papillary Thyroid Cancer Patients after Radioactive Iodine Therapy at the National Cancer Center, Korea

\begin{tabular}{|c|c|c|c|c|c|c|}
\hline \multirow{2}{*}{ Variable } & \multicolumn{6}{|c|}{ No. of metastasized LNs } \\
\hline & 0 & 1 & 2 & 3 & 4 & 5 \\
\hline No. of patients & 295 & 137 & 99 & 66 & 32 & 21 \\
\hline Mean dose of $\mathrm{I}^{131}, \mathrm{GBq}$ & 2.59 & 3.15 & 4.07 & 4.14 & 5.81 & 5.00 \\
\hline Mean 1st sTg, ng/dL & 1.1 & 1.2 & 3.2 & 1.9 & 5.9 & 2.1 \\
\hline Mean last sTg, ng/dL & 0.4 & 0.4 & 0.4 & 1.2 & 3.3 & 1.7 \\
\hline Last sTg $\geq 2 \mathrm{ng} / \mathrm{dL}$ & $2(1)$ & $4(5)$ & $2(4)$ & $3(5)$ & $6(19)$ & $2(17)$ \\
\hline Biochemical incomplete response & $3(1)$ & $3(2)$ & $4(4)$ & $4(6)$ & $2(6)$ & $2(10)$ \\
\hline Structural incomplete response & $3(1)$ & $1(1)$ & $4(4)$ & $1(2)$ & $3(9)$ & $0(0)$ \\
\hline
\end{tabular}


Table 4. Biochemical Outcome of Patients with Metastatic Lymph Node Ratio Less Than 0.4 According to the Number of Metastatic Lymph Nodes, Analyzed Retrospectively from National Cancer Center Database

\begin{tabular}{|c|c|c|c|c|c|c|}
\hline \multirow{2}{*}{ Variable } & \multicolumn{6}{|c|}{ No. of metastatic LNs } \\
\hline & 0 & $\leq 1$ & $\leq 2$ & $\leq 3$ & $\leq 4$ & $\leq 5$ \\
\hline No. of patients & 295 & 432 & 501 & 533 & 541 & 544 \\
\hline Mean dose of $\mathrm{I}^{131}, \mathrm{GBq}$ & 2.59 & 2.78 & 2.92 & 2.96 & 3.00 & 3.00 \\
\hline Mean 1st sTg, ng/dL & 1.1 & 1.2 & 1.4 & 1.4 & 1.4 & 1.4 \\
\hline Mean last sTg, ng/dL & 0.4 & 0.4 & 0.4 & 0.4 & 0.4 & 0.4 \\
\hline Last sTg $\geq 2 \mathrm{ng} / \mathrm{dL}$ & $2(1)$ & $6(2)$ & $7(2)$ & $8(2)$ & $8(2)$ & $8(2)$ \\
\hline Biochemical incomplete response & $3(1)$ & $5(1)$ & $9(2)$ & $11(2)$ & $11(2)$ & $11(2)$ \\
\hline Structural incomplete response & $3(1)$ & $4(1)$ & $5(1)$ & $5(1)$ & $5(1)$ & $5(1)$ \\
\hline
\end{tabular}

Values are expressed as number (\%).

LN, lymph node; sTg, stimulated thyroglobulin.

The number of lymph node metastases is an important prognostic marker for predicting recurrence (Table 3). Based on the number of lymph nodes with metastasis, the frequency of the last positive sTg ( $\geq 2 \mathrm{ng} / \mathrm{dL}$ ) was increased, and four and more lymph nodes with metastasis were strongly correlated with persistent sTg, which suggested that "three or less metastasized lymph nodes" could warrant BCR. From this analysis, the cutoff number of lymph nodes with metastasis to waive RAI should be three.

The number of metastasized lymph nodes is important, although the ratio of metastasized to dissected lymph nodes is also meaningful [9]. In using a ratio of 0.4 , the mean first sTg level and the frequency of persistent $\mathrm{sTg}$ to $>2 \mathrm{ng} / \mathrm{mL}$ were higher in the group with $\leq 0.4$ than in the group with $>0.4$. The structural incomplete response was $7 \%$ in the group of $>0.4$ and $1 \%$ in the group of $\leq 0.4$ (Supplemental Table S3). Moreover, in the group of $\leq 0.4$, the number of metastasized lymph nodes was not critical to predicting persistent $\mathrm{sTg} \geq 2 \mathrm{ng} / \mathrm{mL}$ (Table 4). From these data, we confirmed that the ratio of 0.4 is a key parameter to predict persistent STg after RAI in patients with lowto intermediate-risk of DTC. These analyses yielded the cutoff number and ratio of metastatic lymph nodes and defined the eligible study candidates from among patients with PTC who had low- to intermediate-risk.

The study design has some limitations. Subjects who enrolled in this study would not be assigned to each arm by randomization, but by the patients' preferences. To reduce potential bias, allocation after randomization was attempted in a pilot study; however, the rate of agreement for the random assignment was only $38 \%$. In addition, there are no previous relevant data of the $\mathrm{BCR}$ rate in non-ablated patients with low- to intermediate-risk, and there are potential ethical issues that may interfere with the patients' right to appropriate care. This low rate of agreement can cause severe bias to this study, and the purpose of this study was to evaluate and collect actual data on the efficacy and safety of a treatment; therefore, the non-randomized allocation was selected for this study protocol.

Nevertheless, this study and the IoN trial [10] are the first two prospective trials to assess the actual BCR rate after surgery only or RAI and follow-up patients with low- to intermediate-risk DTC.

\section{CONFLICTS OF INTEREST}

No potential conflict of interest relevant to this article was reported.

\section{ACKNOWLEDGMENTS}

This work was supported by the National Cancer Center grant number 1410650-1,2,3, 1810210-3. The funding authority had no influence on the study design; collection, management, and interpretation of data; writing the report; or decision to submit the report for publication.

\section{AUTHOR CONTRIBUTIONS}

Conception or design: E.K.L., Y.J.L., Y.J.P., K.H.Y. Acquisition, analysis, or interpretation of data: E.K.L., J.H.M., K.H.Y., K. S.K., J.H.L., Y.H., S.W.C., J.J., S.G., D.J.P. Drafting the work or revising: E.K.L., Y.J.L., Y.J.P., K.H.Y. Final approval of the manuscript: E.K.L., Y.J.L., Y.J.P., J.H.M., K.H.Y., K.S.K., J.H.L., S.W.C., J.J., Y.H., S.G., D.J.P. 


\section{ORCID}

Eun Kyung Lee https://orcid.org/0000-0003-0098-0873

Young Joo Park https://orcid.org/0000-0002-3671-6364

\section{REFERENCES}

1. Haugen BR, Alexander EK, Bible KC, Doherty GM, Mandel SJ, Nikiforov YE, et al. 2015 American Thyroid Association Management guidelines for adult patients with thyroid nodules and differentiated thyroid cancer: the American Thyroid Association Guidelines task force on thyroid nodules and differentiated thyroid cancer. Thyroid 2016;26:1-133.

2. Yi KH, Lee EK, Kang HC, Koh Y, Kim SW, Kim IJ, et al. 2016 Revised Korean Thyroid Association management guidelines for patients with thyroid nodules and thyroid cancer. Int J Thyroidol 2016;9:59-126.

3. Mallick U, Harmer C, Yap B, Wadsley J, Clarke S, Moss L, et al. Ablation with low-dose radioiodine and thyrotropin alfa in thyroid cancer. N Engl J Med 2012;366:1674-85.

4. Schlumberger M, Catargi B, Borget I, Deandreis D, Zerdoud $\mathrm{S}$, Bridji $\mathrm{B}$, et al. Strategies of radioiodine ablation in patients with low-risk thyroid cancer. N Engl J Med 2012;366: 1663-73.

5. Chan AW, Tetzlaff JM, Altman DG, Laupacis A, Gotzsche
PC, Krleza-Jeric K, et al. SPIRIT 2013 statement: defining standard protocol items for clinical trials. Ann Intern Med 2013;158:200-7.

6. Ryu CH, Park B, Ryu J, Ryu YM, Jo SA, Lee YJ, et al. Development and evaluation of a Korean version of a thyroidspecific quality-of-life questionnaire scale in thyroid cancer patients. Cancer Res Treat 2018;50:405-15.

7. Dow KH, Ferrell BR, Anello C. Quality-of-life changes in patients with thyroid cancer after withdrawal of thyroid hormone therapy. Thyroid 1997;7:613-9.

8. NCSS. Chapter 210: Non-inferiority tests for the difference between two proportions [Internet]. Kaysville: NCSS; c2020 [cited 2020 Jul 13]. Available from: https://ncsswpengine.netdna-ssl.com/wp-content/themes/ncss/pdf/Procedures/PASS/Non-Inferiority_Tests_for_the_Difference_ Between_Two_Proportions.pdf.

9. Jeon MJ, Yoon JH, Han JM, Yim JH, Hong SJ, Song DE, et al. The prognostic value of the metastatic lymph node ratio and maximal metastatic tumor size in pathological N1a papillary thyroid carcinoma. Eur J Endocrinol 2013;168:219-25.

10. Mallick U, Harmer C, Hackshaw A, Moss L; IoN Trial Management Group. Iodine or Not (IoN) for low-risk differentiated thyroid cancer: the next UK National Cancer Research Network randomised trial following HiLo. Clin Oncol (R Coll Radiol) 2012;24:159-61. 